\title{
HIPERTENSÃO ARTERIAL E INSUFICIÊNCIA CARDÍACA: ANÁLISE CRÍTICA DOS NOVOS FÁRMACOS
}

\author{
ARTERIAL HYPERTENSION AND HEART FAILURE: CRITICAL ANALYSIS OF \\ NEW DRUGS
}

Anderson Wilnes Simas Pereira', Beatriz Castello Branco Miranda², Bruno Wilnes Simas Pereira², Raphael Eltink Trad Coutinho²

\begin{abstract}
RESUMO
A insuficiência cardíaca (IC) permanece como uma das maiores causas de hospitalização e mortalidade dentre todas as doenças, sendo a hipertensão arterial um dos mais importantes fatores de risco para o seu desenvolvimento. Em virtude da relevância e prevalência dessa doença, um número recorde de estudos comparativos, além de novas classes terapêuticas, tem sido desenvolvido nos últimos anos para garantir ao médico maior flexibilidade no tratamento do seu paciente, com a finalidade de frear a progressão do remodelamento ventricular e reduzir a internação hospitalar, bem como os desfechos cardiovasculares. Nesse intuito, o presente artigo avalia diversas meta-análises recentemente publicadas, as quais comparam IECAs aos BRAs e indicam um melhor prognóstico associado aos IECAs na redução de mortalidade total e cardiovascular, provavelmente devido ao efeito adjuvante da bradicinina como cardioprotetor. No entanto, os BRAs ganharam novo destaque a partir de sua associação ao sacubitril, reduzindo eventos cardiovasculares como reinternação por IC e morte cardíaca quando comparados a um IECA. Este artigo de revisão busca realizar uma análise crítica todas as classes de anti-hipertensivos com função na IC, com base na evidência científica mais recente, discorrendo sobre seus mecanismos de proteção cardiovascular e de seus resultados na redução da morbimortalidade cardíaca.
\end{abstract}

Descritores: Insuficiência Cardíaca; Tratamento; Metanálise.

\begin{abstract}
Heart Failure (HF) remains as one of the most important causes of both hospitalization and mortality among all diseases, with hypertension being one of the major factors of risk for its development. As a result of the relevance and prevalence of this disease, a record number of comparative studies, apart from new pharmacological classes, have been developed in the last few years to provide more flexibility to physicians on the process of choosing a proper treatment for patients, for the purpose of slowing ventricular remodeling and reducing hospitalization, as well as cardiovascular outbreaks. Within this context, the present study has examined recently published meta- analysis, which compared ACEi and ARBs, and indicates better prognosis of ACEi in the reduction of all-cause mortality and cardiovascular mortality, probably due to the effect of bradykinin as a cardioprotector. However, it is important to acknowledge that ARBs have gained new emphasis since it was associated with sacubitril, reducing cardiovascular events such as hospitalization for HF and cardiovascular death when compared to ACEi. This current revision article seeks to accomplish a critical analysis of all classes of antihypertensives that act on heart failure by analyzing the most recent scientific evidence, discoursing about its mechanisms of cardiovascular protection and its results in the reduction of cardiovascular mortality.
\end{abstract}

Keywords: Heart Failure; Treatment; Meta-Analysis.

\section{INTRODUÇÃO}

A insuficiência cardíaca (IC) permanece como importante causa de morbimortalidade, tendo sido responsável, apenas no ano de 2012, por 26.694 óbitos e por $21 \%$ de todas as internações decorrentes de quadros cardiovasculares no Brasil. ${ }^{1}$ Como parte importante da fisiopatologia da IC, tem-se o desenvolvimento de alterações estruturais no miocárdio, as quais geram prejuízos no enchimento ventricular e na fração de ejeção (FE). Dentro desse panorama, a hipertensão arterial sistêmica (HAS) apresenta-se como uma das principais etiologias da IC, sendo essencial o controle da pressão arterial para a melhora do prognóstico cardíaco.

1. Centro de Tratamento do Diabético, Hipertenso e Portador de Insuficiência Cardíaca (CADHI) de Cabo Frio, RJ, Brasil.

2. Faculdade de Medicina na Universidade Federal de Minas Gerais, UFMG, Belo Horizonte, MG, Brasil.

Correspondência: Anderson Wilnes Simas Pereira. dr.andersonwilnes@gmail.com

http://dx.doi.org/10.47870/1519-7522/2021280127-34 


\section{FISIOPATOLOGIA: DA HIPERTENSÃO ARTERIAL À INSUFICIÊNCIA CARDÍACA}

O desenvolvimento da IC envolve uma série de fatores complexos e de diferentes etiologias. No caso da IC de etiologia hipertensiva, a disfunção diastólica usualmente é a primeira manifestação da doença. Sua ocorrência se dá a partir de uma sobrecarga crônica da pressão arterial elevada, que conduz a um aumento da massa cardíaca por hipertrofia dos cardiomiócitos, reduzindo o volume sistólico e a distensibilidade cardíaca. Tais alterações comprometem o enchimento ventricular e, por consequência, provocam o desenvolvimento de insuficiência cardíaca com fração de ejeção normal (ICEFEn), definida pela fração de ejeção (FE) maior que $50 \%{ }^{2}$

No entanto, em pacientes com sobrecarga de volume (obesos ou portadores de doença renal crônica), assim como portadores de hipertrofia ventricular esquerda (HVE) que mantém níveis pressóricos elevados, o processo de remodelamento ventricular evolui para dilatação das cavidades cardíacas, o que provoca prejuízo ao débito cardíaco (DC) e ocasiona insuficiência cardíaca com fração de ejeção reduzida (ICFEr), definida por $\mathrm{FE}$ menor que $40 \%$. A elevação de marcadores cardíacos, tais como troponina T de alta sensibilidade e BNP/ pró-BNP, aumentam os riscos dos pacientes com HVE de evoluírem para ICFEr.

Dentro desse contexto, de acordo com diretriz proposta pela European Society of Cardiology, ${ }^{3}$ pacientes com FE entre 40 e 49\% são classificados como portadores de insuficiência cardíaca com fração de ejeção intermediária (ICFEi), definição que engloba tanto os que se encontram na transição da ICFEr para ICFEn após instituição de terapia adequada, quanto aqueles com IC em piora gradual do quadro clínico, progredindo de ICFEn para ICFEr. Tal diferenciação é importante para direcionar a terapia medicamentosa.

\section{ESTÁGIOS DA INSUFICIÊNCIA CARDÍACA}

Atualmente, segundo diretriz da American Heart Association (AHA) de 2013, a insuficiência cardíaca pode ser classificada em:

Estágio A: paciente hipertenso com disfunção diastólica, mas sem alterações estruturais no ventrículo esquerdo.

Estágio B: paciente hipertenso com alterações estruturais na musculatura cardíaca, mas sem sinais e sintomas de IC.

Estágio C: paciente hipertenso com alterações estruturais no miocárdio, sinais e sintomas prévios ou atuais de IC.

Estágio D: paciente hipertenso com alterações cardíacas estruturais e quadro refratário ao tratamento.

\section{METAS PRESSÓRICAS}

As metas pressóricas no tratamento medicamentoso da hipertensão arterial sistêmica (HAS) têm sido abordadas por uma série de importantes estudos internacionais, tais como o estudo HOT, o estudo HYVET e, mais recentemente, o estudo SPRINT.

O estudo SPRINT comparou os benefícios do controle pressórico mais intensivo (PAS $<120 \mathrm{mmHg}$ ) ao controle padrão (PAS < 140mmHg) em pacientes hipertensos com risco cardiovascular elevado. No entanto, foi precocemente interrompido após o grupo com tratamento mais rigoroso da
PA apresentar um índice significativamente menor de desfechos cardiovasculares compostos em comparação ao grupo-controle. A mortalidade em decorrência de acidente vascular cerebral (AVC), infarto agudo do miocárdio (IAM) e IC também foi menor no grupo em tratamento intensivo. Em contrapartida, a ocorrência de eventos adversos, tais como hipotensão arterial, lesão renal aguda, síncope e hipocalemia foi mais prevalente no grupo com tratamento mais rigoroso da PA., ${ }^{4,5}$

Pacientes com doença arterial coronariana (DAC) merecem especial atenção no que tange às metas pressóricas. Nesses pacientes não é recomendado controle pressórico rigoroso (PA $<120 / 70 \mathrm{mmHg}$ ), devido ao aumento do risco de eventos isquêmicos relacionados à lesão miocárdica por hipoperfusão coronariana. A recomendação é a mesma para pacientes idosos, haja visto o risco aumentado de hipotensão postural nessa população. ${ }^{6,7}$

Em decorrência dos resultados clínicos presentes na literatura, há uma tendência em se recomendar controle pressórico inferior a 130/80 mmHg para pacientes com risco cardiovascular elevado, mantendo a pressão arterial superior a 120/70 em pacientes idosos ou com DAC. Pacientes hipertensos estágios 1 e 2 com risco cardiovascular baixo a moderado devem ter como meta pressórica PA <140/90 $\mathrm{mmHg} \cdot{ }^{6-8}$ As metas pressóricas em paciente com IC, por outro lado, permanecem divergindo na literatura. Atualmente, recomenda-se que as metas pressóricas para pacientes com IC sejam semelhantes àquela recomendada para pacientes com elevado risco cardiovascular (PAS entre 120 e $130 \mathrm{mmHg}$ ).

Em pacientes com IC avançada, na qual o quadro de cardiomiopatia dilatada gera comprometimento significativo da fração de ejeção, é comum a ocorrência de PAS baixa mesmo em indivíduos previamente hipertensos. Tal observação pode ser explicada pelo grave comprometimento do ventrículo esquerdo, evento que culmina em reduzido débito cardíaco e hipotensão arterial, o que caracteriza, dessa forma, um quadro conhecido como "hipertensão decapitada". Para esses pacientes, têm-se dificuldade no controle da pressão por introdução terapêutica otimizada para insuficiência cardíaca a partir de fármacos como inibidores da enzima conversora de angiotensina (IECAs), bloqueadores dos receptores de angiotensina (BRAs) e betabloqueadores (BBs). A redução mais intensa da PAS está relacionada com maior taxa de mortalidade, principalmente em pacientes com PAS menor que $100 \mathrm{mmHg}$, em comparação com o grupo de pacientes com nível médio de PAS entre 130 e $139 \mathrm{mmHg}$. Esse fato pode ser justificado pela capacidade do ventrículo esquerdo de manter o DC por meio da manutenção de PAS média mais elevada. ${ }^{2,9-11}$

\section{ESCOLHA DO AGENTE ANTI-HIPERTENSIVO}

A escolha do tratamento anti-hipertensivo adequado, para que este obtenha real eficácia terapêutica, deve levar em consideração alguns parâmetros específicos. Muito além de atingir metas pressóricas, é preciso considerar a proteção adicional dos órgãos-alvo, a preferência por medicamentos com meia vida longa e de dose única diária, os quais aumentam a adesão à terapia vigente, e, não menos importante, a realidade socioeconômica do paciente, especialmente dos que dependem do Sistema Único de Saúde. Tais parâmetros devem sempre ser considerados no momento de escolha do agente hipertensivo, 
o que, no entanto, nem sempre consiste em tarefa fácil, uma vez que os medicamentos mais novos e eficazes nem sempre são acessíveis para a população mais carente.

$\mathrm{O}$ aumento da disponibilidade e variedade das associações fixas (IECA / BRA + BCC, IECA / BRA + diurético, dentre outras menos utilizadas) reduz a polifarmácia e aumenta a adesão ao tratamento. Tais associações já são sugeridas, como terapia inicial e começando com baixas doses, para pacientes em estágio I de hipertensão arterial que tenham riscos de moderado a grave, ou para os pacientes nos estágios II e III. O aumento da dose ou a administração de um terceiro fármaco consistem no próximo passo, o qual é indicado para os casos em que a associação fixa não é suficiente para o controle adequado da pressão arterial. Alguns modelos de tratamento baseados na associação das três classes de anti-hipertensivos (IECA / BRA + diurético tiazídico + BCC) vêm sendo adotados no hipertenso usual. A associação da espironolactona pode ser utilizada como quarta droga nos casos de hipertensão arterial resistente, e os betabloqueadores, por sua vez, podem ser adotados para os casos de hipertonia adrenérgica. ${ }^{12}$

Em relação ao tratamento do paciente hipertenso com disfunção ventricular, o bloqueio do sistema renina-angiotensina-aldosterona (SRAA) é de suma importância para reduzir o remodelamento do ventrículo esquerdo. Meta-análises comparando os IECAs aos BRAs foram recentemente publicadas. Tais estudos indicaram um melhor prognóstico associado ao uso dos IECAs, provavelmente devido ao efeito adjuvante da bradicinina como cardioprotetor. ${ }^{13}$
Os BRAs ganharam, no entanto, novo destaque a partir de sua associação ao sacubitril, reduzindo desfechos cardiovasculares como reinternação por IC e morte cardíaca quando comparados a um IECA. ${ }^{12}$ Betabloqueadores de terceira geração, os quais se destacam por apresentar propriedades vasodilatadoras mediadas por inibição dos receptores alfa ou elevação de óxido nítrico, são importantes no tratamento da disfunção ventricular. A troca do diurético tiazídico por um diurético de alça também é comum após o diagnóstico de IC descompensada, e se justifica pela necessidade de um maior poder diurético para contrabalancear tanto a retenção salina dos membros inferiores, quanto a congestão pulmonar. A espironolactona, apesar de consistir em um diurético fraco e usualmente complementar à terapêutica com diuréticos de alça, exerce importante papel na modulação do SRAA, contribuindo para resultados mais positivos quando considerados os principais desfechos cardiovasculares. ${ }^{14}$

Portanto, o presente artigo destaca que o ajuste fino das terapias medicamentosas e de suas associações é necessário para trazer estabilidade ao quadro clínico dos pacientes com insuficiência cardíaca, principalmente dos que já apresentam disfunção sistólica grave. Hipotensão arterial, retenção hidrossalina, bradicardias e bloqueios atrioventriculares são fatores que devem ser manejados de maneira adequada nesse processo, sobretudo quando associados a fatores não medicamentosos, tais como restrição ao consumo diário de sódio, alcance do peso corpóreo ideal e manutenção do nível de atividade física adequado. (Figura 1)

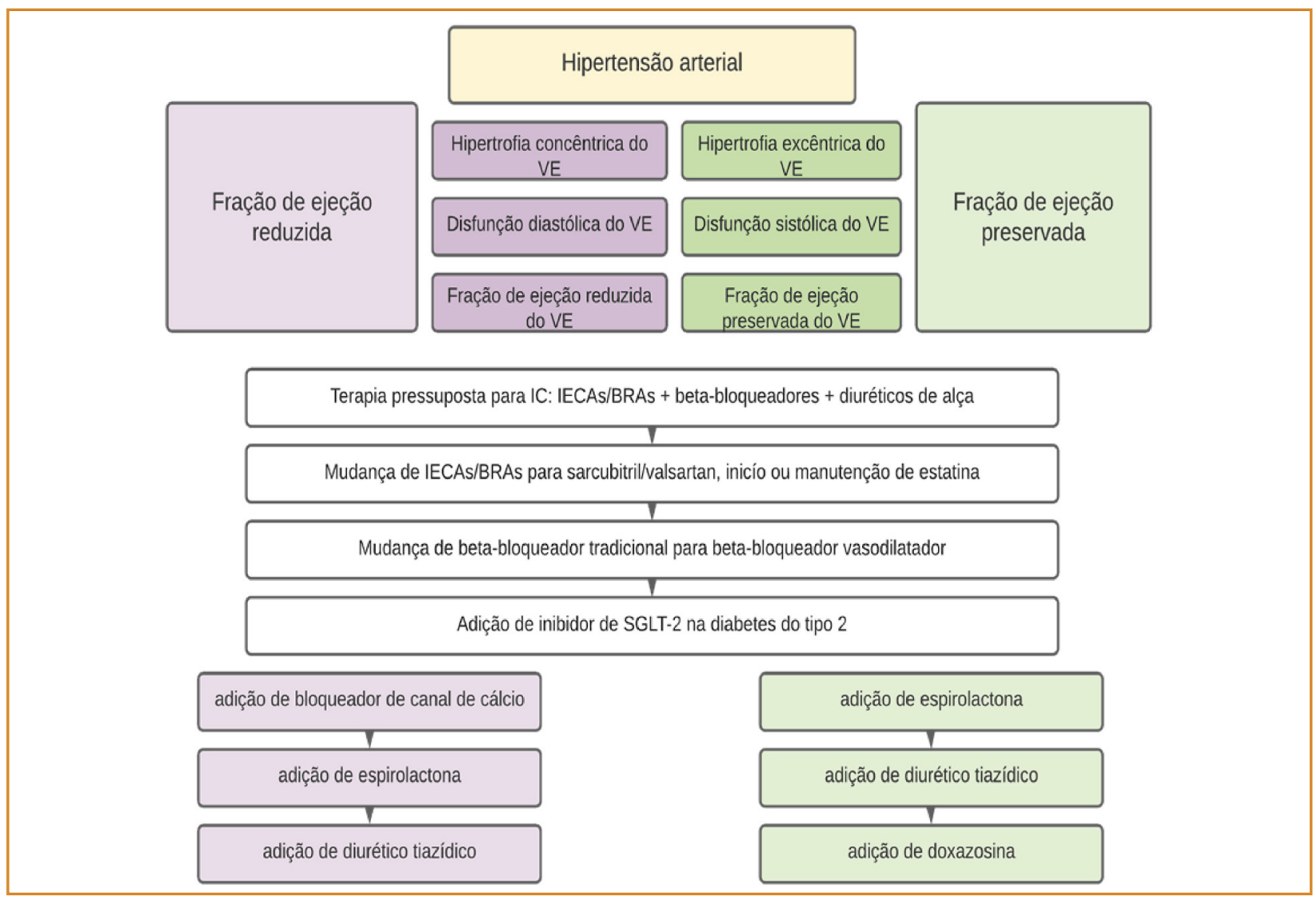

Figura 5. Fluxograma de agentes anti-hipertensivos. IC: insuficiência cardíaca; IECAs: inibidores da enzima conversora de angiotensina; BRAs: bloqueadores de receptores de angiotensina; SGLTs:cotransportador sódio-glicose. Adaptado de Messerli (2017).2 


\section{INIBIDORES DA ENZIMA CONVERSORA DE ANGIOTENSINA}

Em virtude de seu efeito vasodilatador e cardioprotetor, a supressão do SRAA por meio de inibição farmacológica da ECA está associada a melhora do prognóstico clínico do paciente, efetivamente reduzindo o risco de morte em hipertensos. ${ }^{13} \mathrm{~A}$ inibição da enzima conversora apresenta, ao menos, duas vias através das quais exerce seu efeito anti-hipertensivo. Em primeira análise evidencia-se a inibição da formação de angiotensina II e, consequentemente, de seus efeitos vasoconstritores e promotores da disfunção endotelial, com destaque para a redução da biodisponibilidade de óxido nítrico (NO) e aumento da formação de espécies reativas de oxigênio. Um segundo mecanismo se baseia no fato da ECA ser responsável, ainda, pela degradação do peptídeo vasoativo bradicinina. No endotélio, a ativação dos receptores $\mathrm{B} 2 \mathrm{R}$ por meio de ligação com a bradicinina induz a liberação de $\mathrm{NO}$ e prostaglandinas, importantes promotores da vasodilatação e da atividade anti-inflamatória vascular. A eficácia dos IECAs no tratamento de doentes hipertensos, bem como na prevenção de insuficiência cardíaca, está, portanto, centrada tanto em seu duplo efeito vasodilatador, quanto nos efeitos anti-inflamatórios mediados pela bradicinina. ${ }^{15}$

O emprego dos IECAs para o tratamento da hipertensão encontra amplo respaldo na literatura científica. A análise conduzida por L.C. van Vark et al. ${ }^{16}$ demonstrou redução de $10 \%$ na mortalidade de pacientes hipertensos utilizando algum IECA. Estes achados estão em concordância com o estudo meta-analítico desenvolvido por Salvador et al. ${ }^{13}$, cujos resultados apontam para reduções de mortalidade, em decorrência do tratamento com IECA, semelhantes às obtidas pela equipe de van Vark.

A utilização de inibidores da ECA para o tratamento de ICFEn carece de embasamento científico conclusivo. A análise pioneira feita por Emdin et al. ${ }^{17}$, no entanto, indica redução de $12 \%$ no risco de hospitalização para pacientes com ICFEn tratados pela associação de IECA e antagonista dos receptores de aldosterona (ARA), como recomendado pela diretriz de hipertensão para tratamento de ICFEr. ${ }^{12}$ No que se refere ao tratamento da insuficiência cardíaca com fração de ejeção do ventrículo esquerdo menor que 40\%, Emdin et al. atribuem, ainda, reduções amplas na mortalidade geral (26\%), mortalidade cardiovascular (22\%), e hospitalização (30\%), à terapia por bloqueio farmacológico direto do SRAA. ${ }^{16}$ Os dados divulgados indicam resposta aumentada aos inibidores do SRAA na IC com frações de ejeção mais baixas e demonstram a importância dos iECAs, sobretudo no tratamento da ICFEr.

\section{BLOQUEADORES DOS RECEPTORES DE ANGIOTENSINA}

Os bloqueadores dos receptores $\mathrm{AT}_{1}$ de angiotensina II (BRAs) abrangem uma classe de fármacos que, apesar de consolidada, tem sido apontada, em geral, como terapia menos eficaz quando comparada a outros tipos de medicamentos, a exemplo dos IECAs, no tratamento da hipertensão e IC.

A lógica na qual se baseia a utilização dos BRAs está pautada na capacidade do fármaco de bloquear seletivamente os receptores $\mathrm{AT}_{1}$ da angiotensina II (Ang II). Nesse sentido, a evidência científica atualmente disponível sustenta o fato de que a ligação entre Ang II e receptores $\mathrm{AT}_{1}$ atua facilitando ou deflagrando processos endógenos relacionados ao aumento tanto da pressão arterial, quanto da frequência cardíaca, com destaque para vasoconstrição, estímulo à síntese e liberação de aldosterona, aumento da reabsorção de sódio e água nos rins, e potencialização da atividade simpática no sistema nervoso central. Um eventual desequilíbrio no SRAA, comum em pacientes com doença cardiovascular em evolução, pode ser contrabalanceado, dessa forma, pela administração de um BRA. A medicação age bloqueando a ativação dos receptores $\mathrm{AT}_{1}$ e, secundário a este bloqueio, elevando os níveis circulantes de Ang II, as quais tendem a se ligar aos receptores $\mathrm{AT}_{2^{\prime}}$ antagonistas dos receptores $\mathrm{AT}_{1}$, promovendo vasodilatação e redução da pressão arterial. ${ }^{17}$

Os efeitos fisiológicos dos BRAs quando comparados a outras classes medicamentosas, no entanto, não se traduzem em melhoria substancial no prognóstico de pacientes hipertensos. Quanto a isso, L.C. van Vark et al. ${ }^{18}$ indica ausência de eficácia do BRA versus placebo na redução da mortalidade de pacientes hipertensos, enquanto a terapia com IECA foi relacionada a uma redução de $10 \%$ do mesmo fator prognóstico; tais resultados ganharam força com a posterior publicação da análise de Salvador et al. ${ }^{13}$, a qual obteve resultados muito semelhantes.

Em relação ao tratamento tanto da ICFEn, quanto da ICFEr, a literatura científica indica pouca diferença na comparação entre IECA ou BRA quando se considera a melhora do prognóstico para os principais desfechos clínicos, apesar de apontar para resultados ligeiramente mais positivos com o uso de IECAs. ${ }^{19-21}$

Portanto, considerando a evidência científica vigente, o uso de BRA em substituição à terapia com IECA é mais indicado quando o paciente apresenta algum sinal de intolerância ao inibidor da enzima conversora, seja este sintoma uma tosse seca persistente ou, muito raramente, a formação de angioedema. ${ }^{22}$

\section{SACUBITRIL/VALSARTANA}

Recentemente descoberta, a associação farmacológica sacubitril/valsartana (sac/val), medicamento que visa concomitantemente inibir a neprilisina (NEP) e atuar como antagonista dos receptores $\mathrm{AT}_{1}$ da angiotensina II, têm demonstrado surpreendente eficácia no que se refere ao tratamento da insuficiência cardíaca com ou sem hipertensão arterial. A associação de ambos os fármacos em um único medicamento possui benefícios que vão além da redução da polifarmácia.

A inibição da neprilisina, enzima responsável pela degradação de peptídeos natriuréticos, aumenta a disponibilidade desses peptídeos e potencializa seus efeitos vasodilatadores. Os peptídeos natriuréticos promovem vasodilatação por mecanismos diretos e indiretos. A via direta consiste em estimular a guanilato ciclase particulada a produzir GMPc, enquanto as vias vasoativas indiretas se baseiam, sobretudo, em inibir a reabsorção de sódio nos túbulos proximais e distais do néfron, inibir as vias simpáticas responsáveis pela secreção de endotelina e, in vitro, promover a involução do remodelamento cardíaco observado na IC. ${ }^{21}$

A associação de antagonistas do SRAA a inibidores da NEP 
se faz vital para a eficácia do fármaco. A neprilisina catalisa a conversão de angiotensina I em angiotensina (1-7), com a inibição da NEP acarretando o aumento dos níveis de angiotensina II e, consequentemente, de seus efeitos cardiovasculares nocivos. É importante ressaltar, ainda, que a neprilisina, assim como a ECA, contribui para a degradação da bradicinina. $\mathrm{O}$ sacubitril/valsartana deve sua eficácia, dessa forma, tanto aos efeitos cardioprotetores e vasodilatadores da bradicinina, quanto ao aumento da concentração de peptídeos natriuréticos endógenos sem a consequente potencialização dos efeitos relacionados aos níveis aumentados de angiotensina II. ${ }^{21}$

$\mathrm{O}$ sac/val consiste em um medicamento desenvolvido, sobretudo, para atuar na terapêutica da IC. A literatura científica enfatiza, no entanto, que a escolha deste fármaco deve levar em consideração o grau de comprometimento da fração de ejeção ventricular, principalmente no que se refere à ICFEn. Nesse contexto, o ensaio clínico PARAGON-HF, ${ }^{22}$ apesar de concluir que o uso de sacubitril/valsartana não resulta em quedas significativas nas taxas de hospitalização por IC e no número de mortes por causas cardiovasculares, indica potencial benefício da associação quando aplicada a dois grupos específicos: pacientes com fração de ejeção variando entre $45 \%$ e $57 \%$ e mulheres. Tais dados corroboram os achados da análise de Kuno et al., ${ }^{20}$ a qual, por meio de comparações indiretas entre os diferentes tipos de bloqueadores do SRAA, conclui que o sac/val promove redução no número de hospitalizações por IC que, apesar de modesta, não ocorre com outros inibidores do SRAA em pacientes com ICFEn.

O uso do sacubitril/valsartana no tratamento da ICFEr encontra ainda maior respaldo na literatura científica. Nesse sentido, Burnett et al. ${ }^{21}$ obtiveram, por meio de comparação indireta entre resultados de 57 ensaios clínicos, dados que apontam para a combinação sac/val + BB + ARA como a terapia mais eficaz para o tratamento da ICFEr, reduzindo em $63 \%$ a mortalidade em comparação com placebo. A análise de Burnett et al. apresenta, ainda, uma redução de $29 \%$ na mortalidade de pacientes tratados com sac/val versus placebo, desfecho semelhante ao que já havia sido previamente publicado por McMurray et al. ${ }^{23}$ (28\%), e em consonância com a meta-análise desenvolvida por W. Xie et al. ${ }^{24}$, estudo que aponta para a associação entre sacubitril e valsartana como tendo a maior probabilidade de ser a terapia mais eficaz no que se refere à redução da mortalidade e do risco de hospitalização em pacientes com ICFEr.

\section{BETABLOQUEADORES}

Os betabloqueadores não são os anti-hipertensivos mais prescritos no tratamento da hipertensão arterial não complicada sendo, de fato, preteridos pelos iECAs, BRAs, BCCs e diuréticos. No entanto, à medida que o ventrículo esquerdo sofre remodelamento, avançando a cardiopatia hipertensiva e, principalmente, a cardiopatia excêntrica com disfunção sistólica, os benefícios dos betabloqueadores com função vasodilatadora tornam-se inequívocos. O bloqueio, por meio destes, dos receptores adrenérgicos $\beta 1$ gera redução tanto do cronotropismo, quanto do inotropismo cardíaco, agindo, ainda, nas células justaglomerulares renais, nas quais é inibida a liberação de renina, e na readaptação dos barorreceptores, reduzindo a quantidade de catecolaminas liberadas nas sinapses nervosas. ${ }^{25}$
Já o efeito vasodilatador se difere dentre os betabloqueadores: enquanto o carvedilol e o bisoprolol têm sua vasodilatação através do bloqueio dos receptores $\alpha 1$, o nebivolol tem seu efeito vasodilatador no aumento da produção e redução da degradação do óxido nítrico. Quanto à seletividade nos receptores $\beta 1$, nebivolol e bisoprolol são ultrasseletivos, enquanto o carvedilol é pouco seletivo, uma vez que age no bloqueio dos receptores adrenérgicos $\beta 1$ e $\beta 2$, sendo, por esse motivo, inadequado seu uso em pacientes pneumopatas, diabéticos e portadores de disfunção erétil. A ponderação de tais particularidades apresenta vital importância na escolha do betabloqueador ideal para o paciente hipertenso portador de insuficiência cardíaca. ${ }^{25,26}$

Os novos BBs com função vasodilatadora ocasionam acentuada redução da pressão arterial na raiz da aorta, notadamente o nebivolol, o que contribui para a proteção cardiovascular, diferentemente dos betabloqueadores de $1 \underline{a}$ geração como o atenolol. Os BBs de terceira geração são utilizados tanto para ICFEn, por estarem associados à melhora do enchimento ventricular esquerdo através de redução da frequência cardíaca, quanto para ICFEr, em virtude de estarem relacionados à melhora da fração de ejeção e do débito cardíaco por meio do bloqueio dos receptores $\beta 1$, associada ao efeito vasodilatador dessa classe. ${ }^{25,26}$

Em um paciente "virgem do betabloqueador", é recomendado que se inicie a administração do medicamento com baixa dosagem, a qual deve ser aumentada a cada 2 a 4 semanas conforme melhora clínica do paciente, até atingir a dose máxima da medicação, limitada pelo risco de desenvolvimento de hipotensão arterial, bradicardia e bloqueios intracavitários. ${ }^{27}$

A evidência científica mais recente associa o uso de BB, quando associado a outras classes farmacológicas, à redução de internação hospitalar, morte cardiovascular e mortalidade total nos pacientes com insuficiência cardíaca, sendo essa proteção ainda mais exuberante quando são considerados desfechos cardiológicos como fibrilação atrial crônica com elevada resposta ventricular, doença coronariana ou infarto do miocárdio, aneurisma da aorta ou hiperatividade adrenérgica com taquicardia sinusal. Os BBs são utilizados, ainda, em pacientes isquêmicos com insuficiência cardíaca para o tratamento de taquiarritmias do nó atrioventricular e de arritmias ventriculares. $^{27}$

\section{DIURÉTICOS}

Os diuréticos de alça, representados principalmente pela furosemida e pela bumetanida, têm seu mecanismo de ação relacionado à inibição do transportador $\mathrm{NaK} 2 \mathrm{Cl}$, presente na membrana apical das células do epitélio da alça de Henle, nos néfrons. Tais medicamentos atuam inibindo a reabsorção de sódio e cloro na membrana dessas células, o que resulta em aumento da osmolaridade do líquido no túbulo coletor e, consequentemente, maior excreção de líquidos pelo néfron, com impacto na redução do volume de líquido extracelular. São utilizados, principalmente, com o intuito de reduzir sintomas de congestão pulmonar e sistêmica em pacientes com IC e sobrecarga de volume que apresentem hipervolemia e edema. ${ }^{28}$

Os diuréticos tiazídicos, representados principalmente por 
hidroclorotiazida e clortalidona, também são administrados em quadros de descompensação da IC, além de serem amplamente utilizados para o controle pressórico em pacientes hipertensos. Seu mecanismo de ação está relacionado com a inibição da reabsorção dos íons sódio e cloro nos túbulos contorcidos distais do néfron, intensificando a osmolaridade do líquido tubular e, por consequência, aumentando a excreção renal de água. Não são recomendados para pacientes que apresentem doença renal crônica (DRC) com ritmo de filtração glomerular abaixo de $30 \mathrm{~mL} / \mathrm{min}$ sendo, nesses casos, o diurético de alça a melhor escolha para compensação do quadro hipervolêmico. ${ }^{28,29}$

Os diuréticos poupadores de potássio, por sua vez, têm seu uso contínuo mais bem estabelecido na literatura, com efeitos importantes tanto relacionados à mortalidade, quanto à ocorrência de hospitalização, especialmente quando utilizados em associação com IECA/BRA e betabloqueadores em pacientes com ICFEr. O principal medicamento da classe dos diuréticos poupadores de potássio é a espironolactona, a qual possui mecanismo de ação relacionado à inibição competitiva da ligação da aldosterona ao seu receptor, no túbulo contorcido distal, impedindo a reabsorção de sódio e aumentando a reabsorção de potássio no túbulo contorcido distal. ${ }^{30}$

Dessa forma, o uso da espironolactona favorece o controle adequado dos íons séricos do paciente, especialmente do potássio. Esse fármaco é indicado para pacientes que apresentem IC sintomática (classes funcionais II a IV), com disfunção sistólica do ventrículo esquerdo. Estudos mais recentes têm mostrado efeito benéfico da espironolactona no tratamento de ICFEn, principalmente relacionado à redução no número de hospitalizações. ${ }^{30}$

\section{VASODILATADORES DIRETOS (HIDRALAZINA E DINITRATO DE ISOSSORBIDA)}

A hidralazina é um vasodilatador arterial com efeito na musculatura lisa vascular, ${ }^{31}$ atuando na diminuição da resistência sistêmica periférica, redução da pós-carga cardíaca e facilitação do esvaziamento ventricular, contribuindo para o aumento do volume sistólico e da fração de ejeção do ventrículo esquerdo. ${ }^{32} \mathrm{O}$ dinitrato de isossorbida, por outro lado, consiste em um venodilatador com efeito na redução da pré-carga cardíaca, melhorando, pois, o quadro do paciente com insuficiência cardíaca congestiva.

Apesar de alguns ensaios terem demonstrado uma atuação favorável de ambos os medicamentos quando utilizados individualmente, benefícios significativos só foram encontrados com a utilização conjunta dos dois fármacos. Evidências obtidas por meio de ensaios clínicos evidenciaram o potencial da associação medicamentosa na redução da mortalidade e da taxa de internação de pacientes com quadros de IC, sobretudo em estágios III e IV.

O ensaio Veterans Administration Cooperative Vasodilator-Heart Failure Trial I, de 1986, demonstrou a influência do uso conjunto de hidralazina e dinitrato de isossorbida em comparação com a administração de placebo e de prazosina, um bloqueador dos receptores alfa-adrenérgicos, na redução da mortalidade em grupos de pacientes com insuficiência cardíaca de leve a moderada. O número de mortes entre os pacientes diminuiu em 38\% após um ano, 25\% após dois anos e $28 \%$ após o período completo do estudo, de média correspondente a 2,3 anos. $^{33}$

Já o African-American Heart Failure Trial, ocorrido em 2004, foi organizado com o intuito de avaliar a eficácia da associação de hidralazina e dinitrato de isossorbida versus placebo em pacientes negros com insuficiência cardíaca de cllasse III ou IV e que já tivessem tido acesso prévio à terapia medicamentosa. ${ }^{36}$ Após 10 meses de estudo, observou-se que o tratamento de proposto reduziu drasticamente a mortalidade (decréscimo de 43\%) e o risco de hospitalização (decréscimo de $33 \%)^{34}$ o que demonstrou, portanto, que a combinação de hidralazina e dinitrato de isossorbida com o tratamento padrão para ICFEr tem o potencial de aumentar, de forma muito significativa, a taxa de sobrevivência de pacientes afro-americanos com IC avançada.

Diante de tais evidências, depreende-se que as recomendações feitas pela European Society of Cardiology (ESC), em 2016, e pela American Heart Association e American College of Cardiology (AHA/ACCF), em 2013, são acertadas. Recomenda-se o uso da combinação de vasodilatadores diretos em dois casos: o primeiro, em pacientes sintomáticos com ICFEr que não podem tolerar IECAs ou BRAs, ou que possuem contraindicação para esses medicamentos. O segundo, quando os pacientes a serem tratados forem negros e apresentarem ICFEr de classes III ou IV, após a otimização de terapias com IECAs, beta bloqueadores e antagonistas da aldosterona, com o intuito de reduzir o risco de hospitalização e morte desses pacientes.

\section{BLOQUEADORES DOS CANAIS DE CÁLCIO}

Bloqueadores dos canais de cálcio (BCCs), tais como diltiazem, amlodipina e felodipina, consistem em fármacos de primeira linha para o tratamento anti-hipertensivo. Os BCCs consistem em vasodilatadores arteriais capazes de mediar a interrupção do fluxo intracelular de cálcio e, consequentemente, de alterar o tônus vascular, causando vasodilatação, redução na resistência vascular sistêmica e, finalmente, redução da PA. ${ }^{35}$

Suspeita-se, no entanto, que muitos mecanismos de ação dos BCCs contribuam para o desenvolvimento da IC. A longo prazo, a ativação simpática causada pela administração do fármaco parece estar associada à geração de estresse miocárdico, com o acúmulo de catecolaminas, efeito associado à ação do medicamento, sendo prejudicial à performance dos cardiomiócitos. ${ }^{36,37}$

Análises feitas pelo Multicenter Diltiazem Postinfarction Trial Research Group (1988) $)^{38}$ mostram que a administração de diltiazem aumentou, de forma significativa, a mortalidade em pacientes com congestão pulmonar e fração de ejeção do ventrículo esquerdo reduzida após infarto do miocárdio. O estudo também mostrou que a aparição de insuficiência cardíaca foi bem maior em pacientes que utilizavam diltiazem (21\%) na comparação com os indivíduos tratados com placebo (12\%).

A amlodipina e a felodipina, por sua vez, não afetaram de forma significativa a sobrevivência em pacientes com IC e fração de ejeção reduzida. ${ }^{39,40}$ Nesses estudos, no entanto, a administração desses medicamentos foi relacionada a um aumento na incidência de edema pulmonar. 
Portanto, a utilização dos BCCs no tratamento da IC é indicada somente para os casos em que o paciente se mantém hipertenso apesar do tratamento vigente, com o objetivo de alcançar as metas pressóricas, devendo ser preferencialmente empregados a amlodipina ou a felodipina.

\section{INIBIDORES DA SGLT2}

Em decorrência de seu papel na reabsorção de glicose e sódio nos túbulos proximais do néfron, o cotransportador SGLT2 consiste em importante modulador do equilíbrio hemodinâmico e, por isso, objeto de pesquisa no desenvolvimento de terapêuticas para o diabetes melitus tipo II e, mais recentemente, para o tratamento da IC.

As consequências fisiológicas do uso de inibidores da SGLT2 vão além da atividade anti-hiperglicêmica associada ao aumento da glicosúria e do estímulo à natriurese, com a redução do peso corporal e a diminuição da PA consistindo em fatores também importantes para a melhora da hemodinâmica cardiovascular. É muito provável, no entanto, que mecanismos ainda em investigação e mediados, também, pela inibição da SGLT2, tais como diminuição da concentração miocárdica intracelular de sódio, aumento do cálcio mitocondrial e intensificação da produção de cetonas mediada por aumento dos níveis de glucagon, desempenhem papel sinérgico na proteção cardiovascular. ${ }^{41}$

A inibição do SGLT2 tem resultado, dessa forma, em melhoras expressivas do prognóstico de pacientes com ICFEr. Zannad et al. ${ }^{42}$ analisaram os resultados de dois grandes ensaios clínicos orientados para a testagem, em indivíduos com ou sem diabetes mellitus tipo II, dos efeitos relacionados à inibição do SGLT2 no tratamento de pacientes com ICFEr. A comparação estatística desenvolvida pela equipe aponta para reduções de morte cardiovascular (14\%), hospitalização por IC (30\%), e desfechos associados à função renal (38\%), em decorrência do uso de algum inibidor da SGLT2.

Novos ensaios clínicos, no entanto, devem ser desenhados com o intuito tanto de corroborar ou desafiar a aparente eficácia da inibição da SGLT2 na melhora do prognóstico para o tratamento da ICFEr, quanto de verificar a segurança e o efeito a longo prazo do fármaco.

\section{PONTOS-CHAVE}

- ICFEn é a insuficiência cardíaca com FE > 50\%; ICFEr, com FE < 40\%; ICFEi é a insuficiência cardíaca com FE intermediária, entre 40 e 50\%.

- A IC pode ser dividida em estágios A, B, C e D.

- A "hipertensão arterial decapitada" consiste na ICFEr avançada, evoluindo com hipotensão arterial pela falência de bomba cardíaca.

- As meta-análises apreciadas que comparavam os IECAs aos BRAs indicaram um melhor prognóstico associado aos IECAs, sobretudo quanto a redução de mortalidade total e cardiovascular, provavelmente devido ao efeito adjuvante da bradicinina como cardioprotetor.

- Os BRAs ganharam novo destaque a partir de sua associação ao sacubitril, reduzindo desfechos cardiovasculares como reinternação por IC e morte cardíaca quando comparados a um IECA.

- A espironolactona tem sido classicamente utilizada nos pacientes com ICFEr. No entanto, estudos mais recentes têm mostrado efeito benéfico deste medicamento no tratamento de ICFEn, principalmente relacionado à redução no número de hospitalizações.

- A associação hidralasina + nitrato pode ser usada como adjuvante em afrodescendentes após terapêutica para IC padrão estar otimizada.

- Os bloqueadores dos canais de cálcio devem ser utilizados com o intuito de alcançar as metas pressóricas no paciente hipertenso com IC.

- Os inibidores da SGLT2 reduzem internação por IC, morte cardiovascular e desfechos associados à falência renal.

\section{REFERÊNCIAS}

1. BRASIL. Ministério da Saúde. Datasus: mortalidade - 1996 a 2012. Disponível em: http://tabnet.datasus.gov.br/cgi/deftohtm.exe?sim/cnv/ obt10uf.def

2. Messerli FH, Rimoldi SF, Bangalore S. The Transition From Hypertension to Heart Failure: Contemporary Update. JACC Heart Fail. 2017 Aug;5(8):543-551. doi: 10.1016/j.jchf.2017.04.012. Epub 2017 Jul 12. Erratum in: JACC Heart Fail. 2017 Dec;5(12):948.

3. Ponikowski P, Voors AA, Anker SD, et al.; ESC Scientific Document Group. 2016 ESC Guidelines for the diagnosis and treatment of acute and chronic heart failure: The Task Force for the diagnosis and treatment of acute and chronic heart failure of the European Society of Cardiology (ESC)Developed with the special contribution of the Heart Failure Association (HFA) of the ESC. Eur Heart J. 2016 Jul 14;37(27):2129-2200. doi: 10.1093/eurheartj/ ehw128. Epub 2016 May 20. Erratum in: Eur HeartJ. 2016 Dec 30;: PMID: 27206819.

4. De Abreu AP, Da SilvaGV, Drager LF. Análise crítica dos Estudos ACCORD versus SPRINT Resultados e metas pressóricas. Rev. bras. Hipertens. 2016:23(1):2-7.

5. Sprint Research Group; Wright JT Jr, Williamson JD, Whelton PK, et al. A randomized trial of intensive versus standard blood-pressure control. N Engl J Med. 2015; 373(22):2103-16

6. Pires NF, De Faria AP, Modolo R. Hipertensão arterial em pacientes com doença arterial coronariana metas pressóricas. Rev. bras. Hipertens. 2016:23(1):8-15.

7. Malachias MVB, Souza WKSB, Plavnik FL, et al. Sociedade Brasileira de

Cardiologia. 7a Diretriz Brasileira de Hipertensão Arterial. Arq Bras Cardiol [Internet]. 2016;107(3).

8. Thomopoulos C, Parati G, Zanchetti A. Effects of blood pressure lowering on outcome incidence in hypertension. 1. Overview, meta-analyses, and meta-regression analyses of randomized trials. J Hypertens. 2014 Dec;32(12):2285-95. doi: 10.1097/HJH.0000000000000378.

9. Yancy CW, Jessup M, Bozkurt B, etal. 2017 ACC/AHA/HFSA Focused Update of the 2013 ACCF/AHA Guideline for the Management of Heart Failure: A Report of the American College of Cardiology/American Heart Association Task Force on Clinical Practice Guidelines and the Heart Failure Society of America. J Am Coll Cardiol. 2017 Aug 8;70(6):776-803. doi: 10.1016/j. jacc.2017.04.025. Epub 2017 Apr 28.

10. Belyavskiy E, Pieske-Kraigher E, Tadic M.. Blood pressure control in heart failure: Is everything black and white?. JCH. 2019;21(8):1132-34.

11. Comitê Coordenador da Diretriz de Insuficiência Cardíaca, Rohde LEP, Montera MW, Bocchi EA, et al. Diretriz Brasileira de Insuficiência Cardíaca Crônica e Aguda. Arq Bras Cardiol. 2018 Sep;111(3):436-539. Portuguese. doi: 10.5935/abc.20180190. Erratum in: Arq Bras Cardiol. 2019 Jan; 112(1):116.

12. Nielsen PM, Grimm D, Wehland M, Simonsen U, Krüger M. The Combination of Valsartan and Sacubitril in the Treatment of Hypertension and Heart Failure - an Update. Basic Clin Pharmacol Toxicol. 2018 Jan;122(1):9-18. doi: 10.1111/bcpt.12912..

13. Salvador GL, Marmentini VM, Cosmo WR, Junior EL. Angiotensin-converting enzyme inhibitors reduce mortality compared to angiotensin receptor 
blockers: Systematic review and meta-analysis. Eur J Prev Cardiol. 2017 Dec;24(18):1914-1924. doi: 10.1177/2047487317728766. Epub 2017 Sep 1.

14. Xiang Y, Shi W, Li Z, et al. Efficacy and safety of spironolactone in the heart failure with mid-range ejection fraction and heart failure with preserved ejection fraction: A meta-analysis of randomized clinical trials. Medicine (Baltimore). 2019 Mar;98(13):e14967. doi: 10.1097/ MD.0000000000014967.

15. Ancion A, Tridetti J, Nguyen Trung ML, Oury C, Lancellotti P. A Review of the Role of Bradykinin and Nitric Oxide in the Cardioprotective Action of Angiotensin-Converting Enzyme Inhibitors: Focus on Perindopril. Cardiol Ther. 2019 Dec;8(2):179-191. doi: 10.1007/s40119-019-00150-w.

16. Emdin CA, Callender T, Cao J, McMurray JJ, Rahimi K. Meta-Analysis of Large-Scale Randomized Trials to Determine the Effectiveness of Inhibition of the Renin-Angiotensin Aldosterone System in Heart Failure. Am J Cardiol. 2015 Jul 1;116(1):155-61. doi: 10.1016/j.amjcard.2015.03.052.

17. Contreras F, de la Parte MA, Cabrera J, Ospino N, Israili ZH, Velasco $M$. Role of angiotensin II AT1 receptor blockers in the treatment of arterial hypertension. Am J Ther. 2003 Nov-Dec;10(6):401-8. doi: 10.1097/00045391-200311000-00005

18. van Vark LC, Bertrand M, Akkerhuis KM, et al. Angiotensin-converting enzyme inhibitors reduce mortality in hypertension: a meta-analysis of randomized clinical trials of renin-angiotensin-aldosterone system inhibitors involving 158,998 patients. Eur Heart J. 2012 Aug;33(16):2088-97. doi: 10.1093/eurheartj/ehs075.

19. Khan MS, Fonarow GC, Khan H, et al. Renin-angiotensin blockade in heart failure with preserved ejection fraction: a systematic review and meta-analysis. ESC Heart Fail. 2017 Nov; 4(4):402-408. doi: 10.1002/ ehf2.12204

20. Kuno T, Ueyama H, Fujisaki T, Briasouli A, Takagi H, Briasoulis A. MetaAnalysis Evaluating the Effects of Renin-Angiotensin-Aldosterone System Blockade on Outcomes of Heart Failure With Preserved Ejection Fraction. Am J Cardiol. 2020 Apr 15;125(8):1187-1193. doi: 10.1016/j.amjcard.

21. Burnett H, Earley A, Voors AA, et al. Thirty Years of Evidence on the Efficacy of Drug Treatments for Chronic Heart Failure With Reduced Ejection Fraction: A Network Meta-Analysis. Circ Heart Fail. 2017 Jan;10(1):e003529. doi: 10.1161/CIRCHEARTFAILURE.116.003529.

22. Solomon SD, McMurray JJV, Anand IS, et al.; PARAGON-HF Investigators and Committees. Angiotensin-Neprilysin Inhibition in Heart Failure with Preserved Ejection Fraction. N Engl J Med. 2019 Oct 24;381(17):16091620. doi: 10.1056/NEJMoa1908655. Epub 2019 Sep 1. PMID: 31475794.

23. McMurray J, Packer M, Desai A, et al.; PARADIGM-HF Committees and Investigators. A putative placebo analysis of the effects of LCZ696 on clinical outcomes in heart failure. Eur Heart J. 2015 Feb 14;36(7):434-9. doi: 10.1093/eurheartj/ehu455.

24. Xie W, Zheng F, Song X, Zhong B, Yan L. Renin-angiotensin-aldosterone system blockers for heart failure with reduced ejection fraction or left ventricular dysfunction: Network meta-analysis. Int J Cardiol. 2016 Feb 15;205:65-71. doi: 10.1016/j.ijcard.2015.12.010.

25. Do Vale G, Ceron CS, Gonzaga NA, Simplicio JÁ, Padovan J. Three generations of $\beta$-blockers: history, class differences and clinical applicability. Current hypertension reviews. 2019;15(1):22-31.

26. Cruickshank JM. Beta-blockers and heart failure. Indian Heart J. 2010;62(2):101-10.

27. Cruickshank JM. The role of beta-blockers in the treatment of hypertension. In: Hypertension: from basic research to clinical practice. Springer, Cham, 2016. p. 149-166.
28. Ellison DH, Felker GM. Diuretic Treatment in Heart Failure. N Engl J Med. 2017 Nov 16;377(20):1964-1975. doi: 10.1056/NEJMra1703100. Erratum in: N Engl J Med. 2018 Feb 1;378(5):492.

29. Sica DA, Gehr TWB, Frishman WH. Use of Diuretics in the Treatment of Heart Failure in Older Adults. Heart Fail Clin. 2017 Jul; 13(3):503-512. doi: 10.1016/j.hfc.2017.02.006.

30. Pitt B, Pfeffer MA, Assmann SF, et al.; TOPCAT Investigators. Spironolactone for heart failure with preserved ejection fraction. N Engl J Med. $2014 \mathrm{Apr}$ 10;370(15):1383-92. doi: 10.1056/NEJMoa1313731.

31. Knowles HJ, Tian YM, Mole DR, Harris AL. Novel mechanism of action for hydralazine: induction of hypoxia-inducible factor-1alpha, vascular endothelial growth factor, and angiogenesis by inhibition of prolyl hydroxylases. Circ Res. 2004 Jul 23;95(2):162-9. doi: 10.1161/01. RES.0000134924.89412.70.

32. Nyolczas N, Dékány M, Muk B, Szabó B. Combination of Hydralazine and Isosorbide-Dinitrate in the Treatment of Patients with Heart Failure with Reduced Ejection Fraction. Adv Exp Med Biol. 2018;1067:31-45. doi: 10.1007/5584_2017_112.

33. Cohn JN, Archibald DG, Ziesche S, et al. Effect of vasodilator therapy on mortality in chronic congestive heart failure. Results of a Veterans Administration Cooperative Study. N Engl J Med. 1986 Jun 12;314(24):154752. doi: 10.1056/NEJM198606123142404.

34. Taylor AL, Ziesche S, Yancy C, et al.; African-American Heart Failure Trial Investigators. Combination of isosorbide dinitrate and hydralazine in blacks with heart failure. N Engl J Med. 2004 Nov 11;351(20):2049-57. doi: 10.1056/NEJMoa042934. Epub 2004 Nov 8. Erratum in: N Engl J Med. 2005 Mar 24;352(12):1276..

35. Shields DL. Calcium channel blockers as initial therapeutic agents in hypertension: relationship to incident heart failure. Biol Res Nurs. 2014 Jul;16(3):266-77. doi: 10.1177/1099800413494760. .

36. de Champlain J, Karas M, Assouline L, et al. Effects of valsartan or amlodipine alone or in combination on plasma catecholamine levels at rest and during standing in hypertensive patients. J Clin Hypertens (Greenwich). 2007 Mar;9(3):168-78. doi: 10.1111/j.1524-6175.2007.05938.x.

37. Katzung BG,Chatterjee K. Vasodilators and the treatment of angina pectoris. Basic and clinical pharmacology. 2012:7:20-5.

38. Multicenter Diltiazem Postinfarction Trial Research Group. The effect of diltiazem on mortality and reinfarction after myocardial infarction. N Engl J Med. 1988;319(7):385-92. doi: 10.1056/NEJM198808183190701.

39. Elkayam U, Amin J, Mehra A, Vasquez J, Weber L, Rahimtoola SH. A prospective, randomized, double-blind, crossover study to compare the efficacy and safety of chronic nifedipine therapy with that of isosorbide dinitrate and their combination in the treatment of chronic congestive heart failure. Circulation. 1990;82(6):1954-61. doi: 10.1161/01.cir.82.6.1954.

40. Cohn JN, Ziesche S, Smith R, et al. Effect of the calcium antagonist felodipine as supplementary vasodilator therapy in patients with chronic heart failure treated with enalapril: V-HeFT III. Vasodilator-Heart Failure Trial (V-HeFT) Study Group. Circulation. 1997;96(3):856-63. doi: 10.1161/01. cir.96.3.856.

41. Lytvyn Y, Bjornstad P, Udell JA, Lovshin JA, Cherney DZI. Sodium Glucose Cotransporter-2 Inhibition in Heart Failure: Potential Mechanisms, Clinical Applications, and Summary of Clinical Trials. Circulation. 2017 Oct 24;136(17):1643-1658. doi: 10.1161/CIRCULATIONAHA.117.030012. Zannad F, Ferreira JP, Pocock SJ, et al. SGLT2 inhibitors in patients with heart failure with reduced ejection fraction: a meta-analysis of the EMPERORReduced and DAPA-HF trials. Lancet. 2020;396(10254):819-29. doi: 10.1016/S0140-6736(20)31824-9. 